\title{
Efeito da saturação de cor na profundidade de polimerização de resina composta nanohíbrida
}

\author{
Effect of shade in depth polymerization of nanohybrid composite resin \\ Efecto de la saturación del color sobre la profundidad de la polimerización de la resina \\ compuesta nanohíbrida
}

Rafaela Ferreira Damasceno ${ }^{1}$, Lillian Lima e Silva1, Lara Rabelo Aragão², Taira Endi de Flaviano Albuquerque $^{2}$, Vanara Florêncio Passos ${ }^{3}$, Bruna Marjorie Dias Frota de Carvalho ${ }^{2}$, Jiovanne Rabelo Neri $^{1,2 *}$, George Táccio de Miranda Candeiro ${ }^{2}$.

\section{RESUMO}

Objetivo: Avaliar a influência da saturação da cor no grau de conversão (GC) de resina composta nanohíbrida. Métodos: Dez espécimes de resina composta nanohíbrida (5 $\mathrm{mm} \times 5 \mathrm{~mm}$ ) com diferentes saturações de cor (A1, A2, A3 e A3.5) foram obtidas e seccionadas longitudinalmente. Metade de cada espécime foi polido e armazenado a $37^{\circ} \mathrm{C}$ por 24 horas. Para a determinação de GC, as leituras foram realizadas nas profundidades de 0,01 (topo), 1, 2 e 4 milímetros, com um espectrofotômetro micro-Raman. Os procedimentos estatísticos foram realizados com ANOVA a dois critérios e método de Holm-Sidak. A significância estatística foi estabelecida em $p<0,05$. Resultados: Os valores de $G C$ não foram afetados pela profundidade da polimerização até $2 \mathrm{~mm}$, independentemente da saturação da cor da resina composta ( $p>$ $0,05)$. Todos os grupos apresentaram GC significativamente menor na profundidade de $4 \mathrm{~mm}$ quando comparados às demais profundidades $(p<0,05)$. Na profundidade de $4 \mathrm{~mm}$, o grupo A3.5 apresentou menor $G C$ entre os grupos $(p<0,05)$. Conclusão: $A$ saturação da cor das resinas compostas nanohíbridas não interfere na polimerização de até $2 \mathrm{~mm}$ de profundidade. Contudo, cores saturadas, como A3.5, a $4 \mathrm{~mm}$ de profundidade causam redução no GC.

Palavras-chave: Resinas compostas, Polimerização, Conversão, Profundidade.

\section{ABSTRACT}

Objective: To evaluate the influence of color saturation on the degree of conversion (DC) of nanohybrid composite resins. Methods: Ten specimens of a nanohybrid composite resin $(5 \mathrm{~mm} \times 5 \mathrm{~mm})$ with different color saturations (A1, A2, A3 and A3.5) were obtained and sectioned longitudinally. One half of each specimen was polished and was stored at $37^{\circ} \mathrm{C}$ for 24 hours. For DC determination, readings were performed at depths of 0.01 (top), 1, 2 and 4 millimeters, with a micro-Raman spectrophotometer. Statistical procedures were performed with Two-way ANOVA and Holm-Sidak method. Statistical significance was set at $p<0.05$. Results: Degree of conversion values were not affected by the depth of polymerization up to $2 \mathrm{~mm}$, independent of the color saturation of the composite resin $(p>0.05)$. All groups showed a significantly lower DC at depth of $4 \mathrm{~mm}$ when compared to the other depths $(p<0.05)$. At the depth of $4 \mathrm{~mm}$, A3.5 group presented a lower DC between groups $(p<0.05)$. Conclusion: Color saturation of nanohybrid composite resins does not interfere in polymerization up to $2 \mathrm{~mm}$ deep. However, the saturated colors, such as A3.5, at $4 \mathrm{~mm}$ depth cause reduction in DC.

Key words: Composite resins, Polymerization, Conversion, Depth.

1 Universidade de Fortaleza (UNIFOR), Fortaleza - CE.

2 Centro Universitário Christus (UNICHRISTUS), Fortaleza - CE *E-mail: jiovanne@hotmail.com

${ }^{3}$ Universidade Federal do Ceará (UFC), Fortaleza - CE. 


\section{RESUMEN}

Objetivo: Evaluar la influencia de la saturación del color en el grado de conversión (GC) de la resina compuesta nanohíbrida. Métodos: Se obtuvieron diez muestras de resina compuesta nanohíbrida (5 mm x 5 $\mathrm{mm}$ ) con diferente saturación de color (A1, A2, A3 y A3.5) y se seccionaron longitudinalmente. La mitad de cada muestra fue pulida y almacenada a $37^{\circ} \mathrm{C}$ durante 24 horas. Para la determinación de GC, las lecturas se tomaron a profundidades de 0.01 (arriba), 1, 2 y $4 \mathrm{~mm}$ con un espectrofotómetro micro-Raman. Los procedimientos estadísticos se realizaron utilizando ANOVA de dos vías y el método Holm-Sidak. La significación estadística se estableció en $p<0,05$. Resultados: los valores de $G C$ no se vieron afectados por la profundidad de polimerización de hasta $2 \mathrm{~mm}$, independientemente de la saturación de color de la resina compuesta $(p>0.05)$. Todos los grupos presentaron CG significativamente más baja a $4 \mathrm{~mm}$ de profundidad en comparación con las otras profundidades $(p<0.05)$. A una profundidad de $4 \mathrm{~mm}$, el grupo A3.5 presentó el CG más bajo entre los grupos $(p<0.05)$. Conclusión: La saturación de color de las resinas compuestas nanohíbridas no interfiere con la polimerización de hasta $2 \mathrm{~mm}$ de profundidad. Sin embargo, los colores saturados como A3.5 a $4 \mathrm{~mm}$ de profundidad causan reducción de GC.

Palabras clave: Resinas compuestas, Polimerización, Conversión, Profundidad.

\section{INTRODUÇÃO}

A crescente demanda por estética, entre os pacientes, e os avanços dos materiais dentários, nos últimos 50 anos, proporcionaram uma revolução na prática odontológica (FERRACANE JL, 2011). O surgimento de materiais restauradores adesivos e estéticos, como as resinas compostas, possibilitaram a confecção de restaurações imperceptíveis e com grande longevidade, em dentes anteriores e posteriores, sendo uma alternativa ao uso do amálgama de prata e das resinas acrílicas (BORGIA E, et al., 2019; KRÄMER N, et al., 2015). Os compósitos odontológicos são materiais obtidos a partir da mistura de uma matriz polimérica (monômeros dimetacrilato), partículas de carga inorgânicas (vidros radiopacos), um agente de união (silano) e um agente químico fotoiniciador (FERRACANE JL, 2011; KLAPDOHR S e MOSZNER N, 2005). As características das partículas de carga, especialmente o tamanho, determinam os diferentes tipos de resinas compostas existentes no mercado, além de influenciar, diretamente, a lisura superficial e a estética das restaurações (FERRACANE JL, 2011).

Os primeiros compósitos dentários desenvolvidos, chamados de "macropartículas", tinham cargas inorgânicas com aproximadamente $50 \mu \mathrm{m}$ de diâmetro, e eram muito resistentes as tensões, contudo, também apresentavam dificuldade de polimento e manutenção do brilho. Com o objetivo, de melhorar a estética foram formuladas as resinas de "micropartículas", contendo partículas de carga com 40 nm e apresentavam ótimo polimento, porém tinham baixa resistência mecânica (FERRACANE JL, 2011). As resinas compostas nanohíbridas são constituídas por uma mistura de partículas de carga de micropartículas e nanopartículas, com tamanho variando entre 0,04 a 3,0 $\mu \mathrm{m}$ (DE MORAES RR, et al., 2009; TANTHANUCH S e KUKIATTRAKOON B, 2019). Adicionalmente, esses compósitos dentais possuem alta concentração em volume das partículas de carga e presença de pré-polímeros de tamanhos reduzidos (MANOJLOVIC D, et al., 2015; TANTHANUCH S e KUKIATTRAKOON B, 2019). A composição garante a essas resinas compostas excelente polimento, manutenção do brilho, alta resistência à flexão, tração e compressão, fazendo com que sejam classificadas como "universais", ou seja, podem ser utilizadas em dentes anteriores e posteriores (BALDISSERA RA, et al., 2013; LIRA RQN, et al., 2019; ST-PIERRE L, et al., 2019; THOMAIDIS S, et al. 2013).

Por outro lado, a longevidade e o sucesso, dos procedimentos clínicos executados com resinas compostas dependem diretamente do grau de conversão dos monômero sem polímeros (AGUIAR FH, et al., 2011; MAKTABI H, et al., 2018). A polimerização das resinas compostas é uma reação em cadeia iniciada quando a luz, num comprimento de onda entre 400 e $500 \mathrm{~nm}$, correspondente ao espectro azul, atinge a canforoquinona e está reage com uma amina terciária, se transformando em um radical livre (PERIOTTO HA, et al., 2019). Em seguida, a canforoquinona reage com os monômeros resinoso e as duplas ligações de 
carbono são convertidas em ligações simples (PERIOTTO HA, et al., 2019; TAUBOK, 2014). Os monômeros reagirão entre si e formarão uma estrutura com alto peso molecular denominada de polímero (CATELAN A, et al., 2014; FRAUSCHER KE e ILIE N, 2012; PERIOTTO HA, et al., 2019; TAUBOK, TT, et al. 2014). De modo geral, o grau de conversão de monômeros em polímeros das resinas compostas pode ser influenciado por diversos fatores, incluindo a intensidade de luz, o tempo de exposição, o volume do incremento e a saturação da cor (ALSHAAFI MM, 2017; STRYDOM C, 2002).

A resina composta é um material restaurador extremamente versátil e que possui vários tipos de matiz, croma, translucidez e opacidade, possibilitando mimetizar a estrutura dental sadia e assegurar resultados estéticos satisfatórios (ALZRAIKAT H, et al., 2018; MANOJLOVIC D, et al., 2015). As cores da maioria dos compósitos restauradores em uso da Odontologia seguem a escala Vita, que organiza os matizes através das letras A, B, C e D, representando marrom, amarelo, cinza e vermelho, respectivamente (TANOUE N, et al. 2001). A saturação da cor, também conhecida como croma, é representada por números, de forma diretamente proporcional, ou seja, quanto maior a saturação da cor maior será sua numeração (TANOUE N, et al. 2001). Entretanto, existe uma controvérsia quanto o uso de resinas compostas com maior croma, uma vez que pode interferir na profundidade de polimerização (CEFALY DF, et al., 2005). As resinas compostas, com alto croma, possuem maior quantidade de pigmentos na sua composição, que poderia dificultar a penetração da luz na resina composta (OBICI AC, et al., 2004). Alterações no grau de conversão podem causar variações em algumas propriedades físico-mecânicas da resina composta, pois afetam diretamente a densidade de reticulação da cadeia polimérica (STANSBURY JW e DICKENS SH, 2001).

Desta forma, o objetivo do presente estudo foi avaliar o efeito da saturação de cor na profundidade de polimerização de resina composta nanohíbrida. As hipóteses nulas testadas foram: 1) não haverá diferença estatística entre os valores de grau de conversão independente da profundidade do espécime; 2) não haverá diferença estatística nos valores de grau de conversão independente da saturação de cor das resinas compostas.

\section{MÉTODOS}

Para a realização do experimento foi selecionada uma resina composta nanohíbrida de dentina, com diferentes saturações de cor - A1, A2, A3 e A3,5 - (Quadro 1). Foram confeccionados ao todo quarenta espécimes em forma de cilindro, com dimensões de $5 \mathrm{~mm}$ de diâmetro $\times 5 \mathrm{~mm}$ de altura, sendo dez espécimes para cada grupo.

Quadro 1 - Delineamento experimental e composição da resina composta.

\begin{tabular}{|c|c|c|}
\hline Grupos/ Cores & Resina composta & \multicolumn{1}{|c|}{ Composição } \\
\hline A1 & Llis (FGM, Joinville, SC, Brasil) & $\begin{array}{l}\text { Bis-GMA, Bis-EMA, TEGDMA, } \\
\text { canforoquinona, co-iniciador, silano, vidro de } \\
\text { bário-alumino, silicato micronizado, } \\
\text { pigmentos, sílica manométrica. }\end{array}$ \\
\hline A2 & A3 \\
\hline A3,5 & \\
\hline
\end{tabular}

Abreviações: Bis-GMA: Bis-Fenol A di-Glicidil Metacrilato; Bis EMA: Bis-Fenol A di-Glicidil Metacrilato etoxilado; TEGDMA: Trietileno glicol dimetacrilato.

Fonte: Damasceno RF, et al., 2019.

Cada espécime foi obtido a partir da inserção de um único incremento de resina composta no centro da matriz de teflon, com o auxílio de uma espátula (Espátula Millennium dupla № 3045; Golgran, São Caetano do Sul, SP, Brasil). Em seguida, o compósito dental foi acomodado delicadamente com um condensador 
metálico (Calcador Millennium duplo № 3094; Golgran, São Caetano do Sul, SP, Brasil), para evitar a inclusão de bolhas de ar, coberta com uma tira de poliéster de $4 \mathrm{~mm}$ (Maquira, Maringá, PR, Brasil) e uma lamínula de vidro, a qual foi pressionada manualmente por 60 segundos, promovendo o extravasamento do excesso de material. Após a remoção da lamínula de vidro, os espécimes foram polimerizados através da tira de poliéster utilizando uma unidade fotoativadora (Poly Wireless; Kavo, Berlim, Alemanha) com intensidade de $1000 \mathrm{~mW} / \mathrm{cm}^{2}$, durante 40 segundos.

Imediatamente após a fotoativação, os cilindros de resina compostas foram, cuidadosamente, removidos da matriz de teflon e seccionados longitudinalmente com o auxílio de um disco de diamante (Extec modelo 12205; Extec Corp., Enfield, CT, EUA) montado a uma máquina de corte (Labcut 1010; Extec, Enfield, CT, EUA), sob refrigeração abundante, de acordo com a ISO 4049:2000.

As metades de cada cilindro foram fixadas com cera em placas de acrílico. Os espécimes foram polidos com lixa de carbeto de silício № 600 e 1200 acoplada a uma máquina politriz elétrica giratória (Aropol 2V; Arotec, São Paulo, SP, Brasil) (DE CAMARGO EJ, et al., 2009). Os espécimes foram devidamente identificados e, os mesmos, foram armazenados em recipientes opacos por 24 horas, em um ambiente sem iluminação e com temperatura controlada $\left(23 \pm 1^{\circ} \mathrm{C}\right)$, para garantir que o processo de polimerização fosse completamente concluído antes da análise de grau de conversão (LEPRINCE JG, et al. 2012).

Para determinar o grau de conversão, foram realizadas quatro leituras em cada espécime, nas profundidades de 0,01 (topo), 1, 2 e $4 \mathrm{~mm}$ com uma microssonda Raman a laser controlada por computador acoplada a um microscópio óptico com uma objetiva 100 \# e um dispositivo de carga acoplada a um espectrógrafo de pesquisa modular (Xplora, Horiba, Paris, França).

A objetiva de 100 \# aumentou a precisão do feixe, resultando em um tamanho de ponto do laser $\leq 1 \mu \mathrm{m}$. Um laser monocromático de diodo de infravermelho de $638 \mathrm{~nm}$ foi utilizado para induzir o efeito de espalhamento Raman. A faixa espectral utilizada foi de 1500 a $1800 \mathrm{~cm}-1$. Uma calibração de comprimento de onda e intensidade foi realizada com um padrão de silício, usando o sistema de calibração integrado ao software (LabSpec 6; Horiba, Paris, França) antes de cada experimento, seguindo as especificações do fabricante.

O grau de conversão foi calculado com base na redução da intensidade de pico correspondente aos grupos metacrilato $\mathrm{C}=\mathrm{C}$ em $1636 \mathrm{~cm}^{-1}$ e $1608 \mathrm{~cm}^{-1}$ polimerizado $(\mathrm{P})$ em comparação com a amostra não polimerizada (NP), de acordo com a seguinte equação estabelecida por Shin WS, et al. (1993): Grau de conversão (\%) = $(1-\mathrm{P} / \mathrm{NP}) \times 100$

A partir dos dados obtidos, a análise estatística dos resultados foi realizada com o programa SigmaStat 3.5 (Systat Software Inc., San Jose, CA, EUA). Os valores referentes de grau de conversão foram, inicialmente, avaliados com os testes de Shapiro-Wilk e Brown-Forsythe, para analisar a distribuição normal dos dados e a igualdade de variância, respectivamente. Os dados apresentaram distribuição normal, desta forma foi utilizado o teste de Análise de Variância (ANOVA) a dois critérios (saturação de cor da resina composta e profundidade de polimerização). Para as comparações post-roc foi realizado o teste de HolmSidak e o nível de significância adotado foi de $p<0,05$.

\section{RESULTADOS}

Os valores de grau de conversão estão apresentados na Tabela 1. Os resultados foram influenciados pela profundidade de polimerização ( $p<0,01 ; F=86,017)$, mas não pela saturação da resina composta $(p=0,430$; $F=0,933)$ e as interações foram estatisticamente significantes $(p<0,01 ; F=5,970)$.

Os valores de grau de conversão não foram afetados pela profundidade de polimerização até $2 \mathrm{~mm}$, independente da saturação da resina composta $(p>0,05)$. Todos os grupos apresentaram o grau de conversão significativamente menor na profundidade de $4 \mathrm{~mm}$, quando comparado com as demais profundidades $(p<0,05)$. Na profundidade de $4 \mathrm{~mm}$, o grupo com a maior saturação de cor $(A 3,5)$ apresentou menor grau de conversão entre os grupos $(p<0,05)$ 
Tabela 1 - Média dos valores de grau de conversão (em percentual) e desvio padrão, de acordo com a saturação de cor da resina composta e a profundidade de polimerização.

\begin{tabular}{lcccc}
\hline \multirow{2}{*}{ Grupos } & \multicolumn{4}{c}{ Profundidade de polimerização } \\
\cline { 2 - 5 } & Topo & $1 \mathrm{~mm}$ & $2 \mathrm{~mm}$ & $4 \mathrm{~mm}$ \\
\hline $\mathrm{A} 1(n=10)$ & $80,6 \pm 3,6^{\mathrm{Aa}}$ & $84,1 \pm 6,1^{\mathrm{Aa}}$ & $81,9 \pm 6,8^{\mathrm{Aa}}$ & $62,9 \pm 3,1^{\mathrm{ABb}}$ \\
\hline $\mathrm{A} 2(n=10)$ & $80,9 \pm 4,2^{\mathrm{Aa}}$ & $84,8 \pm 3,4^{\mathrm{Aa}}$ & $81,5 \pm 2,1^{\mathrm{Aa}}$ & $68,2 \pm 4,7^{\mathrm{Bb}}$ \\
\hline $\mathrm{A} 3(n=10)$ & $84,5 \pm 2,6^{\mathrm{Aa}}$ & $83,7 \pm 13,0^{\mathrm{Aa}}$ & $85,0 \pm 11,8^{\mathrm{Aa}}$ & $57,84 \pm 5,8^{\mathrm{Ab}}$ \\
\hline $\mathrm{A} 3,5(n=10)$ & $85,4 \pm 4,8^{\mathrm{Aa}}$ & $87,3 \pm 5,2^{\mathrm{Aa}}$ & $88,1 \pm \mathrm{A} 7,8^{\mathrm{Aa}}$ & $41,3 \pm 4,8^{\mathrm{Cb}}$ \\
\hline
\end{tabular}

Legenda: Analisando as colunas, letras maiúsculas semelhantes indicam que não há diferença estatisticamente significante quando comparado os grupos com diferentes saturações de cor de resina composta $(p>0,05)$. Analisando as linhas, letras minúsculas semelhantes indicam que não há diferença estatisticamente significante quando comparadas as profundidades de polimerização de um determinado grupo $(p>0,05)$.

Fonte: Damasceno RF, et al., 2019.

\section{DISCUSSÃo}

As resinas compostas apresentam características que permitem ao cirurgião-dentista realizar restaurações adesivas, funcionais e estéticas (ALZRAIKAT H, et al., 2018; FERRACANE JL, 2011). Adicionalmente, os compósitos odontológicos são amplamente indicados como material restaurador, devido à possibilidade de maior preservação de estrutura dentaria remanescente (ALZRAIKAT H, et al., 2018). Os primeiros compósitos desenvolvidos eram ativados quimicamente (ALSHAAFI MM, 2017; BALDISSERA RA, et al., 2013).

A polimerização das resinas compostas com luz azul foi introduzida pela primeira vez em 1970, e os compósitos dentários eram fotoativados durante 20 a 60 segundos (THIAB SS, et al., 2010). Em 2000, 94\% dos dentistas dos Estados Unidos usaram compósitos fotopolimerizáveis, pois permitiam que ao dentista mais tempo de trabalho e controle sobre o início do processo de polimerização, sendo uma significativa vantagem em comparação aos compósitos quimicamente ativados (KRÄMER N, et al., 2015).

Algumas pesquisas foram realizadas com o objetivo de estabelecer uma correlação entre a qualidade de polimerização das resinas compostas e as suas propriedades mecânicas (CATELAN A, et al., 2014; CEKICNAGAS I, et al., 2010; MAKTABI H, et al., 2018). Para que haja uma polimerização satisfatória dos compósitos dentais e assim, bons resultados clínicos das restaurações, é necessário que ocorra uma elevada conversão de monômeros em polímeros (BAGGIO, 2008).

O processo de polimerização dos materiais resinosos deve ser executado de forma bastante criteriosa, caso seja negligenciado poderá resultar em propriedades químicas, mecânicas e físicas inadequadas (AGUIAR FH, et al., 2011; DA SILVA EM, et al., 2008; MOLDOVAN M, et al., 2019). Falhas na retenção das restaurações, fraturas, manchamentos e respostas pulpares adversas podem estar diretamente relacionadas ao baixo grau de conversão das resinas compostas, comprometendo assim a longevidade destes procedimentos clínicos (DA SILVA EM, et al., 2008; FRAUSCHER KE e ILIE N, 2012, YOON TH, et al., 2002).

O grau de conversão dos monômeros resinosos pode ser medido através de métodos indiretos como o teste de dureza Knoop e Vickers (CEKIC-NAGAS I, et al. 2010; TANTHANUCH S; KUKIATTRAKOON B, 2019). Contudo, é preciso analisar com cautela os resultados dos valores de dureza, uma vez que polímeros com dureza similar podem apresentar diferentes estruturas poliméricas e densidade de ligações cruzadas (SOH MS e YAP AU, 2004). Desta forma, muitos os estudos têm preferido utilizar os testes diretos, como a espectroscopia no infravermelho com Transformada de Fourier e a espectroscopia Raman, para avaliar o grau de polimerização de materiais poliméricos (STANSBURY, JW, DICKENS SH, 2001; PAR M, et al. 2019; SHIN WS, et al. 1993; SOARES LES, et al. 2005). 
A espectroscopia Raman é uma técnica sensível ao modo vibracional das moléculas e, assim como outros métodos espectroscópicos, é frequentemente utilizado para análise do processo de polimerização das resinas odontológicas (PAR M, et al., 2019; PERIOTTO HA, et al., 2019; SOARES LES, et al., 2005). O Raman auxilia na determinação do grau de conversão de monômeros dimetacrilatos em polímeros, com base na redução da altura (ou intensidade) das bandas vibracionais das ligações insaturadas de carbono $(C=C)$, em $1.636 \mathrm{~cm}$ ${ }^{1}$ e $1.608 \mathrm{~cm}^{-1}$, comparado com o espécime não polimerizado, ou seja, o composto com monômero puro (SHIN WS, et al., 1993; SOARES LES, et al., 2005). As vantagens deste método espectroscópico são: (1) analisa amostras de qualquer espessura e geometria; (2) algumas partículas inorgânicas, como a sílica, não interferem significativamente nas leituras; e (3) possui alta a sensibilidade à vibração de moléculas simétricas, do tipo $C=C$, presentes nas resinas de dimetacrilato usadas na Odontologia (SHIN WS, et al., 1993). Por estas razões, a espectroscopia micro-Raman foi utilizada no presente estudo para calcular o grau de conversão das resinas compostas.

Existe um consenso na literatura científica de que a espessura máxima da camada do compósito deveria ser de $2 \mathrm{~mm}$, para que se haja uma polimerização uniforme e adequada (FERRACANE JL, et al., 1986; FRAUSCHER KE e ILIE N, 2013; LEPRINCE JG, et al. 2012; PERIOTTO HA, et al., 2019), corroborando com os achados do presente estudo (Tabela 1). Roberts HW, et al. (2004) observaram que na superfície de topo das resinas compostas, a polimerização é mais eficiente devido ao amplo número de fótons que incidem nessa região. Portanto, para o tratamento de lesões cariosas é recomendada a utilização de uma técnica restauradora inserindo várias camadas de compósito, como até $2 \mathrm{~mm}$ de espessura, para que o grau de conversão de monômeros em polímeros seja adequada (FRAUSCHER KE e ILIE N, 2013).

Por outro lado, alguns autores verificaram que incrementos de compósitos com espessura acima de $2 \mathrm{~mm}$ causaram redução significativa no grau de conversão (FERRACANE JL, et al., 1986; FRAUSCHER KE e ILIE N, 2013; LEPRINCE JG, et al., 2012; ROBERTS HW, et al. 2004). Estes dados estão de acordo com os achados do presente estudo (Tabela 1), e, desta forma, a primeira hipótese nula do trabalho foi rejeitada. À medida que a luz dos aparelhos fotopolimerizadores penetra em profundidades maiores que $2 \mathrm{~mm}$ na resina composta ocorre dispersão dos fótons e perda da potência da luz, prejudicando a polimerização (FRAUSCHER KE e ILIE N, 2013; ROBERTS HW, et al. 2004).

A saturação de cor também desempenha um papel importante na transmissão da luz através das resinas compostas (HYUN HK, et al., 2017; TANOUE N, et al., 2001). Alguns autores observaram que compósitos com saturação de cor mais elevada interferiam significativamente na qualidade da polimerização (FERRACANE JL, et al., 1986; HYUN HK, et al., 2017; KOUPIS SN, et al., 2004; TANOUE N, et al., 2001; THIAB SS, et al., 2010). Hyun HK, et al., (2017) verificaram que compósitos com maior croma exigiam mais energia para a polimerização do que os de croma menor por serem menos translúcidos, ou seja, mais opacos.

A maior quantidade de pigmentos, na composição de resina composta com cores de alto croma, normalmente, pode ser associada com uma polimerização superficial, uma vez que absorve e dispersa mais luz e, assim, reduz a sua penetração na resina composta (HYUN HK, et al., 2017; THIAB SS, et al., 2010). Estes dados estão de acordo com os achados do presente estudo, uma vez que na profundidade de $4 \mathrm{~mm}$, o grupo A3,5 apresentou o menor grau de conversão entre todos os grupos (Tabela 1), e assim, a segunda hipótese nula do trabalho foi rejeitada.

Uma alternativa clínica para minimizar os problemas provenientes da baixa qualidade da polimerização de resinas com elevado croma maior seria aumentar a intensidade e tempo de exposição a luz durante a confecção das restaurações (FERRACANE JL, et al., 1986; HYUN HK, et al., 2017; TANOUE N, et al., 2001). Os fabricantes de aparelhos de fotoativadores afirmam que uma exposição durante 20 segundos proporciona uma a profundidade máxima de polimerização de $2,5 \mathrm{~mm}$, para a maioria das cores de resinas compostas (SHORTALL AC. 2005). Por outro lado, cores mais saturadas como C4 deveriam ser fotativadas durante 30 segundos (SHORTALL AC. 2005). Frauscher KE e llie N (2013) verificaram que para obter uma fotopolimerização de qualidade ao utilizar uma resina composta de cor A3, o incremento deveria ter no máximo $2 \mathrm{~mm}$ de espessura e utilizar o tempo de fotoativação de 20 segundos. Entretanto, Hyun HK, et al., (2017) afirmaram que o grau de conversão foi significativamente maior por um tempo de cura de $20 \mathrm{~s}$ em comparação

REAS/EJCH | Vol.Sup.n.42 | e2875 | DOI: https://doi.org/10.25248/reas.e2875.2020 Página 6 de 9 
com $10 \mathrm{~s}$ em uma profundidade de $2 \mathrm{~mm}$, embora a maioria dos à conversão foi alcançada aos 10 segundos, independentemente da cor dos espécimes.

Os diferentes matizes das resinas compostas também interferem na polimerização (TANOUE N, et al., 2001). Os compósitos dentais com cor marrom-amarelado, B na escala Vita, apresentaram menor profundidade de polimerização quando comparado com espécimes de cor marrom-acinzentado, $C$ na escala Vita (TAKAMIZU M, et al., 1988). Estes dados sugerem que um pigmento amarelo absorve mais luz azul, causando uma redução na polimerização em níveis mais profundos (TAKAMIZU M, et al., 1988).

No presente estudo foi utilizado uma resina composta nanohíbrida somente o matiz A, correspondente ao marrom, sendo uma limitação. O que é observado na literatura cientifica é que a profundidade de polimerização é afetada pela intensidade de pigmentação das resinas compostas cujo o agente fotoiniciador é a canforoquinona (FERRACANE JL, et al., 1986; HYUN HK, et al., 2017; KOUPIS SN, et al., 2004; TANOUE $\mathrm{N}$, et al., 2001; THIAB SS, et al. 2010). Entretanto, atualmente no mercado odontológico foram lançados novos materiais restauradores estéticos com outros agentes fotoiniciadores na sua composição, como por exemplo o óxido bis-alquil fosfínico (BAPO Irgacure), a Ivocerina, o óxido mono-alquil fosfínico (Lucirina TPO) e o 1 fenil-1;2-propanodiona (PPD). Portanto, novos estudos dever ser executados no intuito de verificar se as diferenças de matizes e cromas, também, irão interferir no grau de conversão e na profundidade de polimerização destas novas resinas compostas.

\section{CONCLUSÃO}

Com base nos resultados do presente estudo, é possível concluir que as diferentes saturações de cor das resinas compostas nanohíbridas não interferem no grau de polimerização até $2 \mathrm{~mm}$ de profundidade. Contudo, com $4 \mathrm{~mm}$ de profundidade ocorrem prejuízos significativos na conversão de monômeros em polímeros para todas as cores, principalmente para a mais saturada a A3,5. Portanto durante a confecção de restaurações estéticas, os cirurgiões-dentistas deveriam utilizar incrementos de resinas compostas com espessura máxima de $2 \mathrm{~mm}$, independente da saturação de cor. Este cuidado técnico durante os procedimentos restauradores evitaria redução do grau de conversão das resinas compostas, perda das propriedades físicas-mecânicas, falhas no desempenho dos procedimentos clínicos e prejuízos financeiros para os serviços de Odontologia no âmbito público e privado.

\section{AGRADECIMENTOS}

Os autores agradecem ao Programa de Pós-graduação em Odontologia da Universidade Federal do Ceará (PPGO/UFC), por conceder o uso dos laboratórios e dos maquinários, o que garantiram a execução e publicação do presente estudo.

\section{ACRÔNIMOS E ABREVIAÇÕES}

GC: Grau de conversão

$\mathrm{mm}$ : milímetro

$\mu \mathrm{m}$ : micrômetro

nm: nanômetro

Bis-GMA: Bis-Fenol A di-Glicidil Metacrilato

Bis-EMA: Bis-Fenol A di-Glicidil Metacrilato etoxilado

TEGDMA: Trietileno glicol dimetacrilato

$\mathrm{mW} / \mathrm{cm}^{2}$ : milliwatt por centímetro quadrado

CT: Connecticut 


\section{ISO: International Organization for Standardization}

NP: amostra não polimerizada

$P$ : amostra polimerizada

CA: Califórnia

ANOVA: Análise de Variância

$\mathrm{C}=\mathrm{C}$ : ligações insaturadas de carbono

BAPO: óxido bis-alquil fosfínico

TPO: óxido mono-alquil fosfínico

PPD: 1 -fenil-1;2-propanodiona

\section{REFERÊNCIAS}

1. AGUIAR FH, et al. Effect of different light-curing modes on degree of conversion, staining susceptibility and stain's retention using different beverages in a nanofilled composite resin. J Esthet Restor Dent. 2011; 23(2): 106-114.

2. ALSHAAFI MM. Factors affecting polymerization of resin-based composites: A literature review. Saudi Dent J. 2017; 29(2): 48-58.

3. ALZRAIKAT H, et al. Nanofilled Resin Composite Properties and Clinical Performance: A Review. Oper Dent. 2018; 43(4): E173-E190.

4. BALDISSERA RA, et al. Are there universal restorative composites for anterior and posterior teeth? J Dent. 2013; 41(11) :1027-1035.

5. BORGIA E, et al. Quality and Survival of Direct Light-Activated Composite Resin Restorations in Posterior Teeth: A 5- to 20-Year Retrospective Longitudinal Study. J Prosthodont 2019; 28(1): e195-e203.

6. CATELAN A, et al. Effect of light curing protocol on degree of conversion of composites. Acta Odontol Scand. 2014; 72(8): 898-902.

7. CEKIC-NAGAS I, et al. The effect of irradiation distance on microhardness of resin composites cured with different light curing units. Eur J Dent. 2010; 4(4): 440-446.

8. DA SILVA EM, et al. Relationship between the degree of conversion, solubility and salivary sorption of a hybrid and a nanofilled resin composite. J Appl Oral Sci. 2008; 16(2): 161-166.

9. DE CAMARGO EJ, et al. Composite depth of cure using four polymerization techniques. J Appl Oral Sci. 2009; 17(5): 446-450.

10. DE MORAES RR, et al. Nanohybrid resin composites: nanofiller loaded materials or traditional microhybrid resins? Oper Dent. 2009; 34(5): 551-557.

11. FERRACANE JL. Resin composite-state of the art. Dent Mater. 2011; 27(1): 29-38.

12. FERRACANE JL, et al. Relationship between shade and depth of cure for light-activated dental composite resins. Dent Mater. 1986; 2(2):80-84.

13. FRAUSCHER KE, ILIE N. Degree of conversion of nano-hybrid resin-based composites with novel and conventional matrix formulation. Clin Oral Investig. 2013; 17(2): 635-642.

14. FRAUSCHER KE, ILIE, N. Depth of cure and mechanical properties of nano-hybrid resin-based composites with novel and conventional matrix formulation. Clin Oral Investig. 2012; 16(5): 1425-1434.

15. HYUN HK, et al. Effect of shade, opacity and layer thickness on light transmission through a nano-hybrid dental composite during curing. J Esthet Restor Dent. 2017; 29(5): 362-367.

16. KLAPDOHR S, MOSZNER N. New inorganic components for dental filling composites. Monatsh Chem 2005; 136(1): $21-45$.

17. KOUPIS SN, et al. Curing depth of (polyacid-modified) composite resins determined by scraping and a penetrometer. Dent Mater. 2004; 20(10): 908-914.

18. KRÄMER N, et al. Ten-year Clinical Performance of Posterior Resin Composite Restorations. J Adhes Dent. 2015; 17(5): 433-441.

19. LEPRINCE JG, et al. New insight into the "depth of cure" of dimethacrylate-based dental composites. Dent Mater. 2012; 28(5): 512-520.

20. LIRA RQN, et al. Avaliação do efeito de técnicas de acabamento e polimento na rugosidade superficial de resinas compostas. J. Health Biol Sci. 2019; 7(2):197-203. 
21. MAKTABI H, et al. Factors influencing success of radiant exposure in light-curing posterior dental composite in the clinical setting. Am J Dent. 2018; 31(6): 320-328.

22. MANOJLOVIC D, ET AL. Effect of resin and photoinitiator on color, translucency and color stability of conventional and low-shrinkage model composites. Dent Mater. 2016; 32(2):183-191.

23. MOLDOVAN M, et al. Evaluation of the Degree of Conversion, Residual Monomers and Mechanical Properties of Some Light-Cured Dental Resin Composites. Materials (Basel). 2019; 12(13): E2109.

24. PAR M, et al. The effect of excitation laser power in Raman spectroscopic measurements of the degree of conversion of resin composites. Dent Mater. 2019; 35(9): 1227-1237.

25. PERIOTTO HA, et al. Influence of soft-start curing protocol in the degree of conversion of a nanohybrid resin composite. Rev Odonto Cienc 2018;33(1):49-52.

26. ROBERTS HW, et al. The effect of a translucent post on resin composite depth of cure. Dent Mater. 2004; 20(7): 617622.

27. SHIN WS, et al. Determination of the degree of cure of dental resins using Raman and FT-Raman spectroscopy. Dent Mater. 1993; 9(5): 317-324.

28. SHORTALL AC. How light source and product shade influence cure depth for a contemporary composite. J Oral Rehabil. 2005; 32(12): 906-911.

29. SOARES LES, et al. Monomer conversion of composite dental resins photoactivated by a halogen lamp and a LED: a FT-Raman spectroscopy study. Quím Nova. 2005; 28(2): 229-232.

30. SOH MS, YAP AU. Influence of curing modes on crosslink density in polymer structures. J Dent. 2004; 32(4): 321 326.

31. STANSBURY, JW, DICKENS SH. Determination of double bond conversion in dental resins by near infrared spectroscopy. Dent Mater. 2001; 17(1): 71-79.

32. ST-PIERRE L, et al. Influence of Polishing Systems on Surface Roughness of Composite Resins: Polishability of Composite Resins. Oper Dent. 2019; 44(3): E122-E132.

33. STRYDOM C. Curing lights--the effects of clinical factors on intensity and polymerisation. SADJ. 2002; 57(5): 181186.

34. TAKAMIZU M, et al. Efficacy of visible-light generators with changes in voltage. Oper Dent. 1988; 13(4): $173-180$.

35. TANOUE N, et al. Curing depth of different shades of a photo-activated prosthetic composite material. J Oral Rehabil. 2001; 28(7): 618-623.

36. TANTHANUCH S; KUKIATTRAKOON B. The effect of curing time by conventional quartz tungsten halogens and new light-emitting diodes light curing units on degree of conversion and microhardness of a nanohybrid resin composite. J Conserv Dent. 2019; 22(2): 196-200.

37. TAUBOK, TT, et al. Effect of modulated photo-activation on polymerization shrinkage behavior of dental restorative resin composites. Eur J Oral Sci. 2014; 122(4): 293-302.

38. THIAB SS, et al. The Effect of Shade and Curing Time on Depth of Cure (DOC) in Two Types of Composites, Polymerized with a Halogen Light Cure System. Kufa Med.Journal 2010; 13(1): 168-185.

39. THOMAIDIS S, et al. Mechanical properties of contemporary composite resins and their interrelations. Dent Mater. 2013; 29(8): e132-141.

40. YOON TH, et al. Degree of polymerization of resin composites by different light sources. J Oral Rehabil. 2002; 29(12): 1165-1173. 